\title{
Caracterização química e mineralógica de birnessita natural da Mina do Azul (Província Mineral de Carajás, Brasil) e seu análogo sintético (K-OL-1) obtido pelo método sol-gel
}

\section{(Chemical and mineralogical characterization of natural birnessite from Azul Mine (Carajás Mineral Province, Brazil) and its synthetic analogous (K-OL-1) obtained by sol-gel method)}

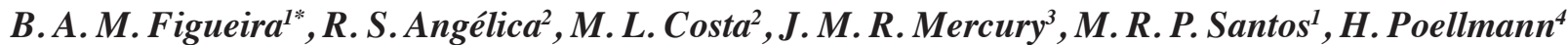 \\ ${ }^{I}$ Universidade Federal do Oeste do Pará, Instituto de Engenharia e Geociências, \\ R. Vera Paz, Campus Tapajós, Santarém, Pará \\ ${ }^{2}$ Universidade Federal do Pará, Instituto de Geociências, R. Augusto Correa, N- 1, Belém, Pará 66075-110 \\ ${ }^{3}$ Instituto Federal do Maranhão, Campus Monte Castelo, Departamento de Química, \\ Av. Getúlio Vargas, $n^{\circ}$ 4, Monte Castelo, S. Luis, MA 65030-005 \\ ${ }^{4}$ Universidade Martin-Luther Wittenberg, Instituto de Geociências, Domstr. 6, D-06108, Halle-Salle, Alemanha \\ *brunoufopa@hotmail.com
}

\begin{abstract}
Resumo
O presente trabalho descreve um estudo comparativo das propriedades química e mineralógica de birnessita natural (BIR-AZ) proveniente da mina do Azul (Província Mineral de Carajás, Brasil) e seu análogo sintético obtido pelo método sol-gel (K-OL-SG). As caracterizações foram realizadas por difração de raios X, espectroscopia FT-Raman, análise térmica (TG-DTA), microscopia eletrônica de varredura e análise química. Os resultados confirmam a presença das fases lamelares com sistema monoclínico, grupo

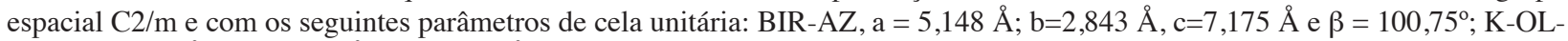
$\mathrm{SG}, \mathrm{a}=5,150 \AA$; $\mathrm{b}=2,846 \AA$, $\mathrm{c}=7,214 \AA$ e $\beta=101,29^{\circ}$. Birnessita possui estabilidade térmica próxima a $600{ }^{\circ} \mathrm{C}$, enquanto seu análogo sintético acima de $900{ }^{\circ} \mathrm{C}$. Bandas obtidas por espectroscopia Raman foram observadas a 635,570 e $410 \mathrm{~cm}^{-1} \mathrm{e}$ atribuídas às deformações $\mathrm{Mn}-\mathrm{O}$ e K-O na estrutura lamelar. O estudo da morfologia dos produtos revela que birnessita se apresenta como folhas tabulares de 1 a $5 \mu \mathrm{m}$, enquanto que o composto sintético possui morfologia em glóbulos $(<1 \mu \mathrm{m})$ dispostos aleatoriamente. Palavras-chave: birnessita natural, Mina do Azul, Província Mineral de Carajás, síntese, sol-gel, caracterização.
\end{abstract}

\begin{abstract}
This paper presents a comparative chemical and mineralogical study of birnessite (BIR-AZ) collected from Azul mine (Carajás Province, Brazil) and its synthetic analogous synthesized by sol-gel method. The products were characterized by X-ray diffraction, FT-Raman spectroscopy, thermal analysis (TG-DTA), scanning electron microscopy (SEM) and ICP-MS techniques. The results obtained confirm the presence of well-defined layer structures having monoclinic system, C2/m space group and the unit cell parameters are: $B I R-A Z, a=5.148 \AA ; b=2.843 \AA, c=7.175 \AA$, and $\beta=100.75^{\circ} ; K-O L-S G, a=5.150 \AA ; b=2.846 \AA, c=7.214 \AA$, and $\beta=101.29^{\circ}$. The birnessite from Azul mine ores shows thermal stability up to $600{ }^{\circ} \mathrm{C}$, whereas its synthetic analogous, above $900^{\circ} \mathrm{C}$. FT-Raman bands at 635, 570 and $410 \mathrm{~cm}^{-1}$ were identified and attributed to the $\mathrm{Mn}$-O and $\mathrm{K}$-O bond deformations in the birnessite structure. SEM images reveals that the manganese oxide mineral consist of platy packed particles of about 1 to 5 um. By contrast, the morphology of the synthetic compound displays randomly arranged globules $(<1 \mu \mathrm{m})$.
\end{abstract}

Keywords: birnessite, Azul Mine, Carajás Province Mineral, synthesis, sol-gel, characterization.

\section{INTRODUÇÃO}

Óxidos de Mn com estruturas em camada (filomanganatos), incluindo birnessita, buserita, lithioforita, vernadita e asbolana, compreendem uma extensa família de minerais de Mn com reconhecida importância científica e tecnológica. Eles são formados a partir da oxidação de soluções ricas em $\mathrm{Mn}^{2+}$, liberado de minerais como biotita, piroxênio, anfibólios, rodocrosita, rodonita, bixbyita, hausmannita, dentre outros. Eles podem ocorrer em diversos ambientes naturais (depósitos minerais de Mn, sedimentos, nódulos do fundo oceânico, verniz do deserto e solos), sendo responsáveis, em grande parte, pela retenção e disponibilidade de elementos traços nestes ambientes, assim como em diversas reações catalíticas, como por exemplo, oxidação de alcoóis e $\mathrm{CO}$, redução de $\mathrm{NO}$ e decomposição de compostos sulfurosos em ambientes marinhos [1-6].

Em relação à birnessita, sua ocorrência foi descrita pela 
primeira vez por Jones e Milne [7], que caracterizaram amostras de óxidos de Mn de um depósito flúvioglacial em Birness (Escócia). Estes autores reportaram a presença do mineral com altos teores de cálcio e sódio, $\left(\mathrm{Na}_{0,7} \mathrm{Ca}_{0,3}\right) \mathrm{Mn}_{7} \mathrm{O}_{14} \cdot 2,8 \mathrm{H}_{2} \mathrm{O}$, e padrões de difração de raios $\mathrm{X}$ típicos de minerais com estrutura lamelar [7]. Posteriormente, outros estudos confirmaram a existência do mineral, porém com potássio, ferro, magnésio, zinco, níquel, cobalto e cobre [8-11]. Segundo Feng et al. [12], que estudaram o seu análogo sintético, birnessita pode ser definida como $\left(\mathrm{A}_{\mathrm{x}}\right)\left[\square_{\mathrm{z}} \mathrm{Mn}^{\mathrm{III}} \mathrm{Mn}^{\mathrm{IV}}{ }_{1-\mathrm{y}-\mathrm{z}}\right] \mathrm{O}_{2} \cdot \mathrm{nH}_{2} \mathrm{O}$, no qual os símbolos A, $\square$, ( ) e [ ] referem-se aos cátions presentes no espaço interlamelar, vazios localizados nos octaedros de $\mathrm{MnO}_{6}$ das camadas, sítios interlamelares e sítios octaédricos dentro das camadas, respectivamente. A posição dos cátions entre as camadas influencia nas propriedades físico-químicas do mineral ou seu análogo sintético, e pode ser em dois sítios principais: i) sítio $\mathrm{A}$, no qual cátions com raio iônico grande estão entre as moléculas de água; ii) sítio B, em que os cátions de raio iônico menor estão acima das moléculas de água [12]. Embora vastamente encontrados na natureza, uma das limitações de caracterização e aplicação deste mineral em áreas comuns como adsorção e catálise, como ocorre com os filossilicatos, está relacionada principalmente ao baixo grau de cristalinidade das fases em amostras naturais, que normalmente encontram-se intercrescidas entre si e misturadas com outros minerais (óxidos de ferro e alumínio, filossilicatos). Variações na composição química também são bastante comuns [5-6, 9]. Tais diversidades resultam na obtenção de amostras com propriedades pouco uniformes, irreprodutíveis e justificam a obtenção de análogos sintéticos destes minerais em laboratório. No presente estudo é apresentada a síntese e caracterização de óxido de manganês com estrutura tipo birnessita, obtido pelo método sol-gel. Um estudo comparativo com seu análogo natural proveniente do depósito de Mn da mina do Azul (Província Mineral de Carajás, Brasil) também é reportado, cuja caracterização química e mineralógica detalhada ainda não foi descrita na literatura.

\section{EXPERIMENTAL}

\section{Obtenção dos compostos lamelares}

O mineral birnessita, proveniente da mina do Azul, província mineral de Carajás (Estado do Pará, norte do Brasil), foi isolado por micropreparação a partir de minérios de óxidos de manganês, grupo predominante de minerais de Mn nesta mina. A fase em estudo foi removida cuidadosamente dos minérios com uma pinça e um microscópio binocular, e foi denominada de BIR-AZ. A fase lamelar com estrutura birnessita foi obtida a partir da metodologia descrita em [13], utilizando glicose como agente redutor, porém com aumento na concentração deste açúcar. Em torno de $50 \mathrm{~mL}$ de uma solução 0,5 mol. $\mathrm{L}^{-1}$ de $\mathrm{KMnO}_{4}$ foram adicionados a $1,7 \mathrm{~mol} \cdot \mathrm{L}^{-1}$ de uma solução de glicose, que após $45 \mathrm{~s}$ formou um gel marrom. Durante $1 \mathrm{~h}$ houve uma constante liberação de água do gel formado, que a cada $10 \mathrm{~min}$ foi drenada para evitar a sua sinérese. $\mathrm{O}$ gel foi seco em estufa a $70{ }^{\circ} \mathrm{C}$ por $12 \mathrm{~h}$ para obtenção de um xerogel marrom. A calcinação deste xerogel por $4 \mathrm{~h}$ a $550{ }^{\circ} \mathrm{C}$ produziu um material cinza-preto que foi lavado, secado a $70{ }^{\circ} \mathrm{C}$ durante $12 \mathrm{~h}$ e codificado como K-OL-SG.

\section{Métodos de caracterização das amostras}

Inicialmente, a caracterização mineralógica foi realizada por difração de raios X usando um difratômetro X'PERT PRO MPD (PW 3040/60), da PANalytical, com goniômetro PW3050/60 (Theta/Theta) e com tubo de raios X cerâmico de anodo de Co (Ka 1,7902 ̊̊), PW3373/00, foco fino longo, $2200 \mathrm{~W}, 60 \mathrm{kV}$. O detector utilizado foi RTMS, X'Celerator. A identificação das fases, assim como os parâmetros de cela unitária, foi obtida a partir do programa X'Pert High Score Plus ${ }^{\circledR}$. Os espectros Raman das amostras foram adquiridos usando um espectrômetro Bruker RFS 100. O laser (Nd:YAG), operando a $\lambda_{0}=1064 \mathrm{~nm}$ serviu como fonte de luz para excitação do espalhamento Raman. O espectro foi registrado na faixa de número de onda de 200 a $800 \mathrm{~cm}^{-1}$ usando uma resolução espectral de $4 \mathrm{~cm}^{-1}$. As curvas de análise termogravimétrica (TG) e análise térmica diferencial (DTA) foram obtidas em um termoanalisador Stanton Redcroft de forno cilíndrico vertical. As análises foram feitas em um cadinho de platina, com aquecimento de $15^{\circ} \mathrm{C} /$ min e variação de temperatura de 25 a $1050{ }^{\circ} \mathrm{C}$.

Para o estudo da morfologia dos materiais obtidos por microscopia eletrônica de varredura (MEV), as amostras foram pulverizadas e dispersas em suportes impregnados com grafite e metalizadas com ouro. O instrumento utilizado foi um microscópio LEO-Zeiss, $430 \mathrm{Vp}$, em condições de análise utilizando imagens secundárias obtidas a $20 \mathrm{kV}$, com distância de trabalho de $11 \mathrm{~mm}$. A composição química da amostra BIR-AZ foi determinada em laboratório comercial (ACME, Canadá). O procedimento de rotina 4A (ICP-MS) foi empregado para a determinação dos elementos maiores após fusão de $0,2 \mathrm{~g}$ da amostra com metaborato/tetraborato de lítio, seguido de digestão com ácido nítrico diluído. A perda ao fogo (LOI) foi obtida pela diferença de massa da amostra antes e após calcinação a $1000{ }^{\circ} \mathrm{C}$ por $4 \mathrm{~h}$. Para a amostra sintética, K-OL-SG, utilizou-se a metodologia descrita em [13]. Uma massa de $0,1 \mathrm{~g}$ foi dissolvida em 50 $\mathrm{mL}$ de água régia e posteriormente diluída em $500 \mathrm{~mL}$ de água deionizada. A determinação dos elementos $\mathrm{K}$ e Mn foi realizada por espectrofotometria de absorção atômica de chama.

\section{RESULTADOS E DISCUSSÃO}

Os padrões de difração de raios $\mathrm{X}$ das amostras de birnessita (BIR-AZ) e seu análogo sintético (K-OL-SG) são mostrados na Fig. 1. Os picos mais intensos próximos a 7 e $3.5 \AA$ foram identificados e se referem aos planos O0l, comuns em compostos de óxidos de Mn com estrutura birnessita, sistema monoclínico e grupo espacial $C 2 / m$ (PDF 42-1317). Também se observa nas amostras um baixo grau 
de cristalinidade, conforme pode ser deduzido pela largura à meia altura dos picos $00 l$, bem como da pouca definição das reflexões na faixa de 10 a $60^{\circ}(2 \theta)$. Esta baixa cristalinidade, segundo Post e Veblen [14], pode estar relacionada às posições dos cátions $\mathrm{K}^{+}$e moléculas de água presentes no espaço interlamelar. Os picos localizados em 6,86, 4,91, 3,10 e 2,39 ̊̊ na amostra natural (BIR-AZ) podem ser atribuídos aos planos (110), (200), (310) e (211) da fase vernadita (PDF 15-0604), que segundo Chukhrov et al. [5] e Post [6] é uma variedade de birnessita desordenada no empilhamento das folhas ao longo da direção $\boldsymbol{c}$. É possível que outros minerais estejam presentes, em baixas concentrações (ou amorfos), porém não detectados por DRX. Os parâmetros de cela unitária dos compostos estudados são mostrados na Tabela I e estão de acordo com os dados da literatura.

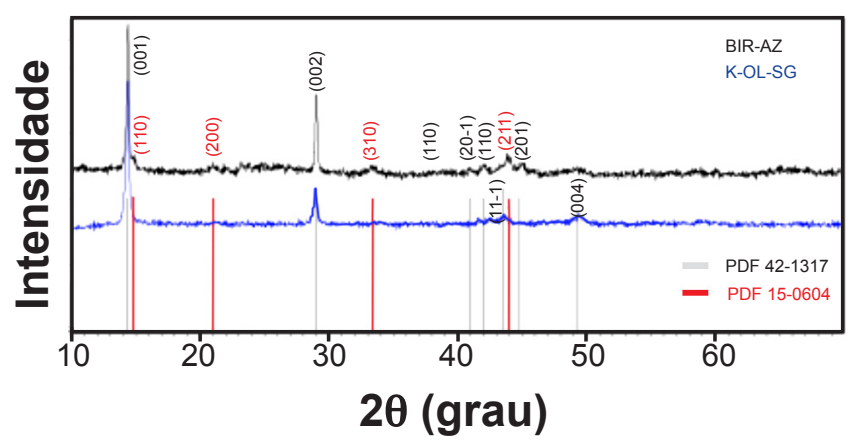

Figura 1: Difratogramas de raios X de K-OL-SG e BIR-AZ. [Figure 1: XRD patterns of $\mathrm{K}-\mathrm{OLS}-\mathrm{SG}$ and BIR-AZ.]

Tabela I - Comparação das constantes de retículo de K-birnessita monoclínica (BIZ-AZ e K-OL-SG) com dados da literatura.

[Table I - Unit cell parameters of BIR-AZ and $\mathrm{K}-\mathrm{OL}-\mathrm{SG}$ compared to literature data.]

\begin{tabular}{ccccc}
\hline & PDF & PDF & BIR-AZ & KOL-SG \\
& $1497-087$ & $42-1317$ & & \\
\hline a $(\AA)$ & 5,149 & 5,15 & 5,148 & 5,15 \\
b $(\AA)$ & 2,843 & 2,844 & 2,843 & 2,846 \\
c $(\AA)$ & 7,176 & 7,159 & 7,175 & 7,214 \\
$\beta(\AA)$ & 100,176 & 100,64 & 100,75 & 101,29 \\
\hline
\end{tabular}

A composição química das amostras BIR-AZ e K-OLSG é apresentada na Tabela II. Como pode ser observado, o óxido de manganês $(\mathrm{MnO})$ apresenta o maior teor nas amostras, com 72,3\% para BIR-AZ e 76,8\% para K-OL-SG, praticamente todo relacionado à fase lamelar anteriormente descrita. Em menor quantidade, o óxido de potássio foi identificado em BIR-AZ (2,43\%), assim como em K-OL-SG $(6,27 \%)$. Outros óxidos maiores também foram observados na amostra natural, tais como de ferro $(2,54 \%)$ e alumínio $(2,56 \%)$, que podem estar presentes na estrutura em camada. Todos os outros óxidos estão em concentrações abaixo de
Tabela II - Composição química dos elementos principais, expressa na forma de óxidos (\%), e traços (ppm).

[Table II - Chemical composition of major and trace elements of BIR-AZ and $\mathrm{K}-\mathrm{OL}-\mathrm{SG}$.]

\begin{tabular}{|c|c|c|c|c|c|}
\hline $\begin{array}{l}\text { Elementos } \\
\text { maiores } \\
(\mathrm{ppm})\end{array}$ & BIR-AZ & K-OL-SG & $\begin{array}{c}\text { Elementos } \\
\text { traços } \\
(\mathrm{ppm})\end{array}$ & BIR-AZ & K-OL-SG \\
\hline $\mathrm{SiO}_{2}$ & 0,72 & $<0,01$ & $\mathrm{Ba}$ & 1201 & $<0,01$ \\
\hline $\mathrm{TiO}_{2}$ & $<0,01$ & $<0,01$ & $\mathrm{Zn}$ & 848 & $<0,01$ \\
\hline $\mathrm{Al}_{2} \mathrm{O}_{3}$ & 2,56 & $<0,01$ & $\mathrm{Ni}$ & 676 & $<0,01$ \\
\hline $\mathrm{Fe}_{2} \mathrm{O}_{3}$ & 2,54 & $<0,01$ & $\mathrm{Cu}$ & 575 & $<0,01$ \\
\hline $\mathrm{MnO}$ & 72,3 & 76,8 & $\mathrm{~Pb}$ & 122 & $<0,01$ \\
\hline $\mathrm{CaO}$ & 0,2 & $<0,01$ & Co & 303 & $<0,01$ \\
\hline $\mathrm{MgO}$ & $<0,01$ & $<0,01$ & $\mathrm{Sr}$ & 354 & $<0,01$ \\
\hline $\mathrm{Na}_{2} \mathrm{O}$ & $<0,01$ & $<0,01$ & & & \\
\hline $\mathrm{K}_{2} \mathrm{O}$ & 2,43 & 6,27 & & & \\
\hline $\mathrm{P}_{2} \mathrm{O}_{5}$ & 0,28 & $<0,01$ & & & \\
\hline LOI & 18,3 & 12,1 & & & \\
\hline Total & 99,36 & 95,17 & & & \\
\hline
\end{tabular}

$1 \%$, como $\mathrm{SiO}_{2}, \mathrm{TiO}_{2}, \mathrm{MgO}, \mathrm{Na}_{2} \mathrm{O}, \mathrm{CaO}$ e $\mathrm{P}_{2} \mathrm{O}_{5}$. Em relação aos elementos traços, destaca-se a presença dos metais bário (1201 ppm), zinco (848 ppm), níquel (676 ppm), cobre (575 ppm), chumbo (122 ppm), cobalto (303 ppm) e estrôncio (354 ppm). Estes resultados confirmam a importante função de birnessita na retenção e liberação de metais em ambientes geoquímicos [1, 5-9]. Com base na estrutura lamelar de birnessita e no raio iônico dos cátions, pode-se estabelecer que os cátions $\mathrm{K}^{+}(1,33 \AA), \mathrm{Ca}^{2+}(0,99 \AA)$ e $\mathrm{Ba}^{2+}$ $(1,35 \AA$ A $)$ estão localizados no espaço interlamelar (sítio A) ou adsorvidos na superfície do mineral, já que não podem substituir os cátions $\mathrm{Mn}^{3+}(0,65 \AA)$ e $\mathrm{Mn}^{4+}(0,53 \AA)$ que estão nos octaedros formando as camadas.

Os espectros dos produtos lamelares em K-OL-SG e BIR-AZ foram obtidos por espectroscopia Raman e são apresentados na Fig. 2. Eles são caracterizados por duas bandas em torno de 635 e $733 \mathrm{~cm}^{-1}$. Estas bandas são diagnósticas de birnessita monoclínica com grupo espacial $\mathrm{C} 2 / \mathrm{m}$ e correspondem aos estiramentos simétricos $n_{2}$ e $n_{3}$ da ligação Mn-O nos "blocos de construção" de $\mathrm{MnO}_{6}$ ao longo da folha octaédrica [15-18]. As bandas próximas a 405 e 375 $\mathrm{cm}^{-1}$ no espectro de K-OL-SG, assim como em 410 e 237 $\mathrm{cm}^{-1} \mathrm{em}$ BIR-AZ, podem ser assinaladas às vibrações das ligações metal-oxigênio que estão no espaço interlamelar, como por exemplo $\mathrm{K}^{+}, \mathrm{Ba}^{2+}, \mathrm{Zn}^{2+}, \mathrm{Ni}^{2+}$, etc. $[14,19,20]$.

A Fig. 3 mostra as curvas de TG/DTA do comportamento térmico dos compostos estudados na faixa de 30 a $1050{ }^{\circ} \mathrm{C}$. $\mathrm{O}$ composto lamelar sintético (K-OL-SG) apresentou duas reações exotérmicas a 75 e $190{ }^{\circ} \mathrm{C}$ com perda de massa total de $7,47 \%$, que corresponde à perda aproximada de 6,5 mol de $\mathrm{H}_{2} \mathrm{O}$ (teórico), com os 2,5 mol de moléculas de $\mathrm{H}_{2} \mathrm{O}$ restantes, sendo liberados no intervalo entre 200 e $900{ }^{\circ} \mathrm{C}$. Birnessita natural também apresentou os mesmos eventos exotérmicos, porém em intervalos de temperatura diferentes. 


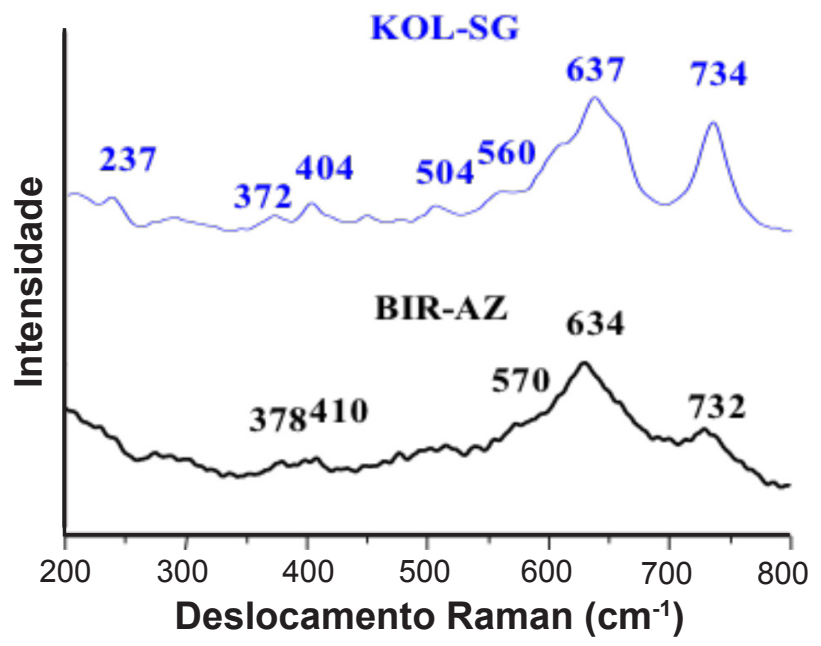

Figura 2: Espectros FT-Raman de BIR-AZ e K-OL-SG. [Figure 2: FT-Raman spectra of BIR-AZ and K-OL-SG.]
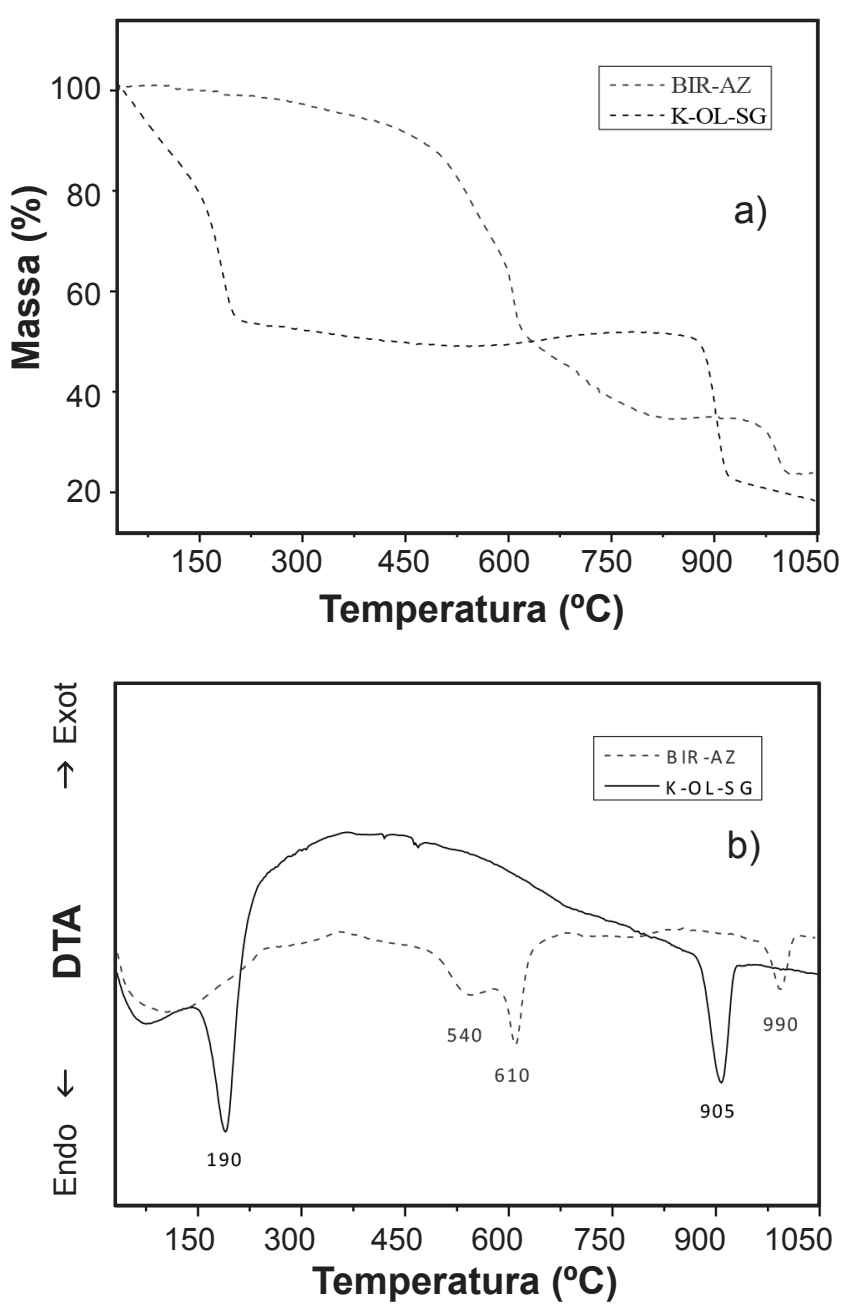

Figura 3: Curvas de TG (a) e DTA (b) a $15^{\circ} \mathrm{C} / \mathrm{min}$ de birnessita natural (BIR-AZ) e seu análogo sintético (K-OL-SG).

[Figure 3: TG (a) and DTA (b) curves at $15{ }^{\circ} \mathrm{C} / \mathrm{min}$ of natural birnessite (BIR-AZ) and its synthetic analogous (K-OL-SG).]
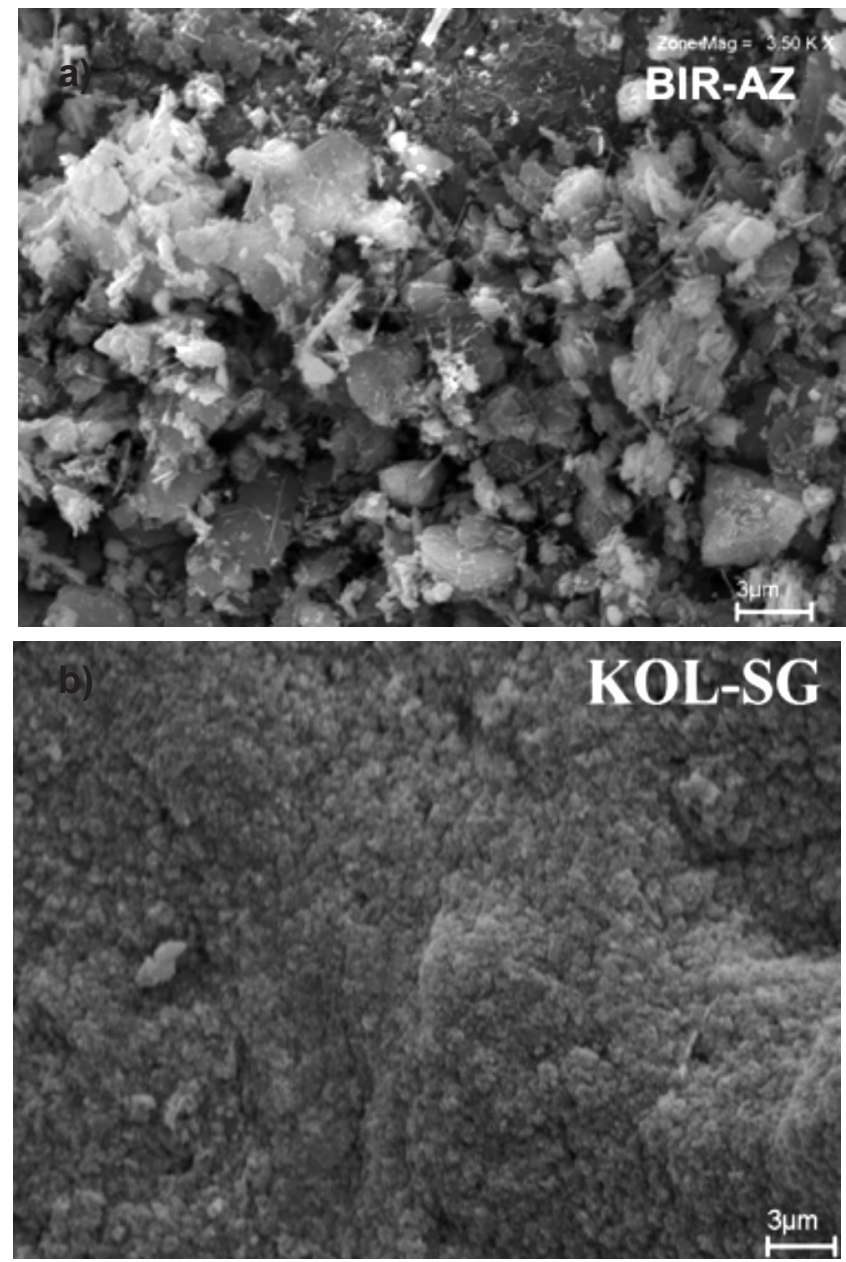

Figura 4: Micrografias obtidas por microscopia eletrônica de varredura das amostras BIR-AZ e K-OL-SG.

[Figure 4: SEM micrographs of BIR-AZ and K-OL-SG.]

O primeiro ocorreu a $110{ }^{\circ} \mathrm{C}$ e o segundo próximo a $545{ }^{\circ} \mathrm{C}$ com perda total de massa de $3 \%$, o que representa a liberação de $5 \mathrm{~mol}$ de moléculas de água nesta temperatura. Os 4 mol restantes foram liberados em intervalos relativamente curtos $\left(545\right.$ a $\left.590{ }^{\circ} \mathrm{C}\right)$. Acima de $600{ }^{\circ} \mathrm{C}$, a curva de DTA de BIR-AZ revela um intenso pico exotérmico com perda de massa de 2,5\% relacionada à completa destruição do composto lamelar natural e formação de $\mathrm{Mn}_{2} \mathrm{O}_{3}$. Evento que também foi observado em K-OL, porém em um intervalo de temperatura próximo a $910{ }^{\circ} \mathrm{C}$ (perda de massa de 4,65\%), revelando maior estabilidade térmica do produto lamelar obtido em laboratório.

A morfologia dos produtos foi investigada por microscopia eletrônica de varredura e é mostrada na Fig. 4. $\mathrm{Na}$ amostra natural (BIR-AZ), foram observados cristais tipo tabulares pseudo-hexagonais com tamanho médio em torno de 1 a $5 \mu \mathrm{m}$. A forma pseudo-hexagonal do produto natural corrobora com os resultados de birnessita sintética descrita por Cai et al. [21], porém difere da morfologia vermiforme de birnessita descrita tanto por Glover [9], como por Brown et al. [10]. Do produto sintético (K-OL-SG), notou-se a presença de glóbulos dispersos aleatoriamente com tamanho de cristais inferiores a $1 \mu \mathrm{m}$. Ching et al. [22] também 
reportaram a mesma morfologia, porém de um produto tipo birnessita sintetizado pelo método sol-gel não aquoso.

\section{CONCLUSÕES}

Birnessita natural da Província Mineral de Carajás foi extraída com sucesso de amostras de minérios de óxidos de Mn através de procedimentos simples de micropreparação. Os resultados por difração de raios X e espectroscopia Raman comprovaram a presença deste mineral, que teve um padrão similar à fase obtida em laboratório pelo método sol-gel (K-OL-SG). Birnessita mostrou uma composição química rica em ferro, potássio e alumínio, com morfologia em placas, típica de minerais lamelares, e estabilidade térmica próxima a $550{ }^{\circ} \mathrm{C}$. Por outro lado, o seu análogo sintético manteve a estrutura em temperatura bem mais elevada (em torno de $900{ }^{\circ} \mathrm{C}$ ). É bem provável que a diferença de cátion no espaço interlamelar dos produtos aqui descritos influenciou de forma incisiva nas propriedades estruturais (parâmetro de cela unitária), térmica e morfológica destes compostos.

\section{AGRADECIMENTOS}

Os autores agradecem ao CNPq pelo auxílio financeiro.

\section{REFERÊNCIAS}

[1] R.G. Burns, V.M. Burns, Marine Manganese Deposits, $1^{\text {st }}$ Ed., Elsevier Oceanography Series, Amsterdam (1979) 188.

[2] B.M. Tebo, B.G. Clement, G.J. Dick, K.J. Murray, D. Parker, R. Verity, S.M. Webb, Annu. Rev. Earth Palnet Sci. 32 (2004) 287.

[3] S.M. Webb, B.M. Tebbo, J.R. Bargar, Amer. Miner. 90
(2005) 1342.

[4] R.M. Mackenzie, Z. Pflanzenernährung Bodenkunde. 131 (1972) 221.

[5] F.V. Chukhrov, A.I. Gorshkov, E.S. Rudnitskaya, V.V. Beresovskaya, A.V. Sivtsov, Clay Clay Miner. 28 (1980) 346.

[6] J.E. Post, Proc. Nat. Acad. Sci. 96 (1999) 3447.

[7] L.H.P. Jones, A.A. Milne, Min. Mag. 31 (1956) 283.

[8] A.A. Levinson, Amer. Miner. 47 (1962) 790.

[9] E.D. Glover, Amer. Miner. 62 (1977) 278.

[10] F.H. Brown, A. Pabst, D.L. Sawyer, Amer. Miner. 56 (1971) 1057.

[11] C. Frondel, U.B. Marvin, J. Ito, Amer. Miner. 45 (1960) 871.

[12] Q. Feng, H. Kanoh, K. Ooi, J. Mater. Chem. 9 (1999) 319.

[13] S. Ching, D.J. Petrovay, M.L. Jorgensen, S.L. Suib, Inorg. Chem. 36 (1997) 883.

[14] J.E. Post, D.R. Veblen, Amer. Mineral. 75 (1990) 477.

[15] C. Julien, M. Massot, R. Baddour-Hadjean, S. Franger,

C. Bach, Solid State Ionics 159 (2003) 345.

[16] C.M. Julien, M. Massot, C. Poisignon, Spect. Acta Part A. 60 (2004) 689.

[17] A. Ogata, S. Komaba, R. Baddour-Hadjean, J.P. PereiraRamos, N. Kumagai, Electrochim. Acta 53 (2008) 3084.

[18] A. Dias, R.G. Sá, M.C. Spitale, M. Athayde, V.S.T. Ciminelli, Mat. Res. Bull. 43 (2008) 1528.

[19] D.P. Dubal, D.S. Dhawal, R.R. Salunkhe, C.D. Lokhande, J. Alloys Compd. 496 (2010) 370.

[20] X. Liu, Q. Chu, X. Wang, X. Zhang, Q. Li, Inorg. Chem. 50 (2011) 2049.

[21] J. Cai, J. Liu, L.S. Suib, Chem. Mater. 14 (2002) 2071.

[22] S. Ching, S.M. Hughes, T.P. Gary, E.J. Welch, Microporous Mesoporous Mater. 76 (2004) 41.

(Rec. 14/09/2014, Rev. 26/10/2015, Ac. 10/12/2015) 